\title{
Fabry-Perot Imaging of the Seyfert 2 Galaxy NGC 2273: Preliminary Report
}

\author{
V. Golev \\ Department of Astronomy, University of Sofia, 5 James Bourchier st., \\ $B G-1126$ Sofia, Bulgaria
}

T. Bonev

Institute of Astronomy, Bulgarian Academy of Sciences, 72 Tsarigradsko chaussee blvd., BG-178 $\$$ Sofia, Bulgaria

K. Jockers

Max-Planck-Institut for Aeronomy, D-37189 Katlenburg-Lindau, Germany

and I. Yankulova

Department of Astronomy, University of Sofia, 5 James Bourchier st., $B G-1126$ Sofia, Bulgaria

Abstract. Narrow-band images of the ringed Seyfert 2 galaxy NGC 2273 = Mark 620 taken with tunable Fabry-Perot interferometer are presented. Some preliminary conlusions are outlined.

\section{Introduction}

We present new narrow-band images of the ( $\left.R^{\prime}\right) \mathrm{SB}(\mathrm{rs})$ a type ringed Seyfert 2 galaxy NGC 2273 = Mark 620 (van Driel \& Buta, 1991) taken with tunable Fabry-Perot (TFP) interferometer. NGC 2273 was observed with the 2-m Ritchey-Chretien-coudé reflector of the Bulgarian National Astronomical Observatory (BNAO) at Mount St. Spirit near Rozhen, Rodopa mountains. The Fabry-Perot images were taken with the Focal Reducer of the Max-PlanckInstitute for Aeronomy (FR MPAe). This instrument was designed by K. Jockers (1992) originally for cometary/planetary studies but since 1991 it has been successfuly used for observations of active galactic nuclei.

\section{Observations and Data Reduction}

We have used a TFP with gap of $32 \mu \mathrm{m}$ which gives a free spectral range $\Delta \lambda \sim 60 \AA$ and a spectral FWHM of Airy function $\delta \lambda \sim 3.5 \div 4.0 \AA$. As detector an EEV $576 \times 385$ CCD type P8603/B with quantum efficiency of $\sim 0.52$ at $\lambda 6500 \AA$ was used. The telescope/FR MPAe configuration and the CCD's square $22 \mu \mathrm{m}$ pixels provide an image scale of $0.8 \mathrm{px}^{-1}$ for an unvignetted field 
of $\sim 7 \times 5$ arcmin at an effective focal ratio $\mathrm{f} / 2.86$. This scale is quite well adapted to the typical seeing conditions of BNAO $\left(2^{\prime \prime} \div 2.5\right.$ FWHM $)$.

Several pairs of on-line and off-line TFP images were obtained in November 1991 around the emission lines of $[\mathrm{O} \mathrm{I}] \lambda 6364,[\mathrm{~N} \mathrm{II}] \lambda 6548$, and $\mathrm{H} \alpha$, as well as in the light of the highly excitated coronal emission lines [Fe VII] $\lambda 6601$ and $[\mathrm{Fe} x] \lambda 6374$. The processing of the Fabry-Perot data follows procedures described by Bonev \& Jockers (1991).

Also, Gunn $g, r$ and $i$ broad-band, and usual interference filter narrow-band images in the light of $\mathrm{H} \alpha+[\mathrm{N}$ II] and [O III] $\lambda 4959$ lines were taken at the end of 1992 to have the opportunity to check our data with those of other authors.

\section{Results}

Narrow-band interference filter images isolating $\mathrm{H} \alpha+[\mathrm{N}$ II] and [O III] emission lines well agree with the images published by Pogge (1989). The high contrast of the tunable Fabry-Perot allows us to have better detection of an extended emission-line region around the galactic nucleus. Also, weak (and uncertain) traces of $[\mathrm{Fe} \mathrm{x}] \lambda 6374$ coronal emission in the circumnuclear region of Mark 620 aligned with the observed radio axis were detected.

The Gunn 8 - Gunn i colour map reveals a complex structure of reddened dusty matter inside the inner galaxy ring. This, together with the prominent far-infrared features of NGC 2273 and its extraordinary $\mathrm{H}_{2}$ richness (Krugel et al., 1990), probably explains the fueling of the accretion onto the central nuclear compact object.

Detailed results will be published elsewhere.

Acknowledgments. Our research was partly supported by the Bulgarian National Scientific Foundation grant under contract No. F-109/1991 with the Bulgarian Ministry of Education and Sciences and by the Deutsche Forschungsgemeinschaft Aktenzeichen 436 BUL 113/61.

Images were processed on a DEC-PC, granted by ESO in the framework of the Central \& Eastern Europe Programme under contract A-03-031.

This research has made use of the NASA/IPAC Extragalactic Database (NED) which is operated by the Jet Propulsion Laboratory, California Institute of Technology, under contract with the National Aeronautics and Space Administration.

\section{References}

Bonev, T., \& Jockers, K. 1991, in Proc. of the 3rd ESO/ST.ECF Data Analysis Workshop, G. Warmels \& P. Grossboel, ESO: Garching bei München, p.29

Jockers, K. 1992, Astronomische Gesellschaft Abstr. Ser. No. 7, P 108, 184

Krugel, E., Steppe, H., \& Chini, R. 1990, A\&A, 229, 17

Pogge, R. W. 1989, ApJ, 345, 730

van Driel, W. \& Buta, R.J. 1991, A\&A, 245, 7 